\title{
Mid-infrared coherent sources and applications: introduction
}

\author{
Majid Ebrahim-Zadeh ${ }^{1, *}$ and Konstantin Vodopyanov ${ }^{2}$ \\ ${ }^{1}$ ICFO-The Institute of Photonic Sciences, 08860 Barcelona, Spain \\ ${ }^{2}$ University of Central Florida, CREOL-The College of Optics \& Photonics, University of Central Florida, Orlando, Florida 32816-2700, USA \\ *Corresponding author: majid.ebrahim@icfo.es
}

Received 24 October 2016; posted 24 October 2016 (Doc. ID 279463); published 28 October 2016

\begin{abstract}
This feature issue, based on the OSA Topical meeting on Mid-Infrared Coherent Sources (MICS), focuses on the most recent advances in mid-infrared to $\mathrm{THz}$ science and technology, from materials to laser sources and applications. (๑) 2016 Optical Society of America
\end{abstract}

OCIS codes: (000.1780) Conferences, lectures, and institutes; (000.2190) Experimental physics.

http://dx.doi.org/10.1364/JOSAB.33.00MIC1

The mid-infrared (mid-IR) spectral range covering wavelengths from $\sim 2 \mu \mathrm{m}$ up to $\mathrm{THz}$ has become a region of increasing interest due a wide variety of emerging scientific and technological applications. The region covers important atmospheric windows and is rich in spectroscopic fingerprints of numerous molecules. Many harmful gases, air, water and soil contaminant, as well as components of human breath and biological tissue have strong absorption fingerprints in this region. This makes the mid-IR spectrum highly important for a variety of applications, including environmental monitoring, atmospheric transmission, breath analysis, minimally invasive surgery, food quality control, spectroscopy, and imaging. The timely progress in mid-IR technology is, therefore, vital for the development of modern diagnostic tools for novel environmental, chemical, biological, and medical applications.

A critical component in the advancement of mid-IR technology is the development of novel laser sources with continually evolving performance capabilities with regard to wavelength coverage, optical power and efficiency, temporal, spectral, and spatial quality, and output stability, in increasingly compact, practical, portable, miniaturized design, and in all time scales from continuous-wave to ultrafast femtosecond domain. This, in turn, relies on progress in material science and the development of new crystalline solid-state and fiber laser gain media, novel semiconductor materials and structures, and new nonlinear crystals for wavelength conversion into the mid-IR. The steady progress in materials and laser sources over the past decade has led to major advances in mid-IR technology, also paving the way for new applications in various areas. This feature issue includes some of the most recent advances in mid-IR science and technology, from materials to laser sources and applications. The feature brings together the latest developments in solid-state and fiber lasers, nonlinear frequency conversion and parametric sources, quantum cascade lasers, as well as the novel applications of mid-IR in trace gas detection, spectroscopy, and imaging.

We hope this feature issue will provide a useful reference on mid-IR sources and applications and will stimulate further research in this important research field. 